\title{
Pineal Parenchymal Cell Neoplasm
}

National Cancer Institute

\section{Source}

National Cancer Institute. Pineal Parenchymal Cell Neoplasm. NCI Thesaurus. Code C6965.

A neoplasm arising from the pineocyte, a cell with photosensory and neuroendocrine functions. It may be composed of mature elements or primitive, immature cells. The cellular composition determines the biological behavior and clinical outcome. Three types are recognized: pineoblastoma, pineocytoma, and pineal parenchymal tumor of intermediate differentiation (Adapted from WHO.) 